\title{
Is there a potential role of fecal microbiota transplantation in the treatment of inflammatory bowel disease?
}

\author{
Chang Soo Eun \\ Department of Internal Medicine, Hanyang University Guri Hospital, Guri, Korea
}

\section{Article: Fecal microbiota transplantation for refractory Crohn's disease (Intest Res 2017;15:244- 248)}

The exact pathogenesis of IBD, including CD and UC, is not clear. However, it has been accepted that IBD results from inappropriate and ongoing immune reactions to specific components of the intestinal microbiota in a genetically susceptible host, triggered by various environmental factors. ' Several human and animal studies have shown that dysbiosis, an altered intestinal microbial profile, is associated with IBD, and that the intestinal microbiota seems to play a crucial role in the development of this disease. Although most current therapies focusing on suppression of the immune system (e.g., corticosteroids, immunosuppressants, and biologics) have been effective in treating IBD, a considerable proportion of patients have still been refractory to these treatments. Considering the pathophysiological role of the intestinal microbiota, targeting the microbiota might be an attractive therapeutic approach for IBD. However, treatments manipulating the microbial imbalance of the intestines, including antibiotics, probiotics, and prebiotics, have been mostly discouraging so far.

As an alternative therapeutic strategy, altering the dysbiotic intestinal microbial community through fecal microbiota transplantation (FMT) has been proposed and investigated in the treatment of IBD. FMT involves the infusion of feces (the entire intestinal microbiota) from healthy donors into

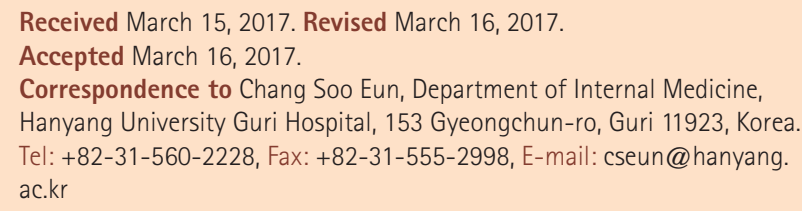

Financial support: None. Conflict of interest: None. the intestinal tract of recipient patients. It has been shown to be highly efficacious for refractory and recurrent Clostridium difficile infection, with more than $90 \%$ of cure rates. ${ }^{2}$ The success of FMT in treating patients with $C$. difficile infection has suggested that FMT could be effective in other diseases associated with dysbiosis such as IBD.

Several studies exploring the efficacy of FMT in patients with IBD have been reported, but the results are equivocal. Regarding UC, a systematic review of four case series that included 27 patients found that $24 \%$ of patients achieved clinical remission. ${ }^{3}$ In 2015, two randomized controlled trials (RCTs) of FMT in patients with UC were published, which reported contradictory results. ${ }^{4,5}$ One study by Moayyedi et al. ${ }^{4}$ that randomized 75 patients with UC to receive FMT (50 $\mathrm{mL}$ enema, from an unrelated healthy donor) or placebo (50 $\mathrm{mL}$ water enema) once weekly for 6 weeks demonstrated a statistically significant benefit in the FMT group, with clinical and endoscopic remission at 7 weeks in comparison with outcomes in the placebo group (24\% vs. $5 \%$, respectively). ${ }^{4}$ On the contrary, a second study by Rossen et al. ${ }^{5}$ that randomized 48 patients with UC to receive FMT (via nasogastric tube, healthy donor) or placebo (autologous fecal microbiota) at 0 and 3 weeks showed no statistically significant difference in remission at 12 weeks (30\% vs. 20\%, respectively). Very recently, another RCT by Paramsothy et al. ${ }^{6}$ that randomized 85 patients with UC to receive FMT ( $150 \mathrm{~mL}$ enema, blended stool from 3 to 7 unrelated donors) or placebo ( $150 \mathrm{~mL}$ enema) 5 days per week for 8 weeks, demonstrated a statistically significant benefit in the FMT group, with clinical (steroid-free) and endoscopic remission at 8 weeks in comparison with outcomes in the placebo

\footnotetext{
๑ Copyright 2017. Korean Association for the Study of Intestinal Diseases. All rights reserved.

This is an Open Access article distributed under the terms of the Creative Commons Attribution Non-Commercial License (http://creativecommons.org/licenses/by-nc/4.0)

which permits unrestricted non-commercial use, distribution, and reproduction in any medium, provided the original work is properly cited.
} 
group ( $27 \%$ vs. $8 \%$, respectively). These results suggest the importance of treatment intensity and multiple donors for each FMT infusion. ${ }^{6}$ In contrast, regarding $C D$, a systematic review of four case series that included 38 patients found that $60.5 \%$ of patients achieved a clinical response. ${ }^{3}$ Of note, there are no RCT data assessing FMT in patients with CD.

However, most of these studies have been performed in Western countries. Compared with Western populations, Asian patients with IBD have distinct genetic and ethnic backgrounds, as well as different environmental features such as diet and intestinal microbial variation. ${ }^{7}$ A recent Japanese, open-label, non-randomized study on 10 patients with UC failed to show the clinical efficacy of FMT (single infusion via colonoscopy, related donor). ${ }^{8}$

In this issue of Intestinal Research, Bak et al. ${ }^{9}$ reported a case of refractory CD treated with a single FMT infusion. A 16-year-old female patient diagnosed as having CD 1 year ago, showed a disease course fluctuating between remission and relapse on receiving treatment with steroids, an immunosuppressant, and an anti-tumor necrosis factor (anti-TNF) agent. The authors tried to treat this patient with refractory CD with FMT. FMT was performed via the upper gastrointestinal tract, using $250 \mathrm{~mL}$ of fecal suspension from a family donor, which was delivered into the second portion of the duodenum through the working channel of a gastroduodenoscope. Fortunately, the patient achieved clinical remission with a single FMT infusion and maintained this remission state with an anti-TNF agent and immunosuppressant. This case report, which first demonstrated the efficacy of FMT in a Korean patient with refractory $\mathrm{CD}$, provides important insight into the treatment options available for these patients in Korea. However, as the authors described, this is just a case report with a possibility of an anecdotal experience. Unfortunately, fecal microbial analysis was not performed to evaluate the effect of FMT on intestinal microbial change in this patient with refractory CD.

FMT might be a promising therapeutic option in patients with IBD. However, before FMT can be considered as a treatment of IBD in clinical practice, several critical issues should be resolved. Whether FMT has a definite efficacy in patients with IBD, especially in those with CD, remains unclear. Indications and timing of FMT in IBD, donor characteristics (e.g., related vs. unrelated, single vs. multiple), most effective FMT protocols (e.g., route of administration, fresh vs. frozen stool, treatment intensity and duration, infusion volume, antibiotics pre-treatment, bowel preparation), and long-term safety and efficacy should be evaluated through additional, welldesigned, large-scale RCTs.

\section{REFERENCES}

1. Yi JM, Kim TO. Epigenetic alterations in inflammatory bowel disease and cancer. Intest Res 2015;13:112-121.

2. van Nood E, Vrieze A, Nieuwdorp M, et al. Duodenal infusion of donor feces for recurrent Clostridium difficile. N Engl J Med 2013;368:407-415.

3. Colman RJ, Rubin DT. Fecal microbiota transplantation as therapy for inflammatory bowel disease: a systematic review and meta-analysis. J Crohns Colitis 2014;8:1569-1581.

4. Moayyedi P, Surette MG, Kim PT, et al. Fecal microbiota transplantation induces remission in patients with active ulcerative colitis in a randomized controlled trial. Gastroenterology 2015;149:102-109.e6.

5. Rossen NG, Fuentes S, van der Spek MJ, et al. Findings from a randomized controlled trial of fecal transplantation for patients with ulcerative colitis. Gastroenterology 2015;149:110-118.e4.

6. Paramsothy S, Kamm MA, Kaakoush NO, et al. Multidonor intensive faecal microbiota transplantation for active ulcerative colitis: a randomised placebo-controlled trial. Lancet 2017;389:1218-1228.

7. Ng WK, Wong SH, Ng SC. Changing epidemiological trends of inflammatory bowel disease in Asia. Intest Res 2016;14:111119.

8. Mizuno S, Nanki K, Matsuoka K, et al. Single fecal microbiota transplantation failed to change intestinal microbiota and had limited effectiveness against ulcerative colitis in Japanese patients. Intest Res 2017;15:68-74.

9. Bak SH, Choi HH, Lee J, et al. Fecal microbiota transplantation for refractory Crohn's disease. Intest Res 2017;15:244-248. 